Source: Tajfard, M., Latiff, L. A., Rahimi, H. R., Moohebati, M., Hasanzadeh, M., Emrani, A. S., Esmaeily H., Taghipour A., Mirhafez, S. R., Ferns, G. A., Mardan-Nik, M., Mohammadzadeh, E., Avan, A., Hanachi P., and GhayourMobarhan, M. (2017). Serum concentrations of MCP-1 and IL-6 in combination predict the presence of coronary artery disease and mortality in subjects undergoing coronary angiography. Molecular and Cellular Biochemistry, 435(1-2), 37-45. https://doi.org/10.1007/s11010-017-3054-5

\title{
Serum concentrations of MCP-1 and IL-6 in combination predict the presence of coronary artery disease and mortality in subjects undergoing coronary angiography.
}

Tajfard $M^{1,2}$, Latiff L.A ${ }^{2}$., Rahimi H.R ${ }^{3}$., Moohebati $M^{4}$., Hasanzadeh $M^{4}$., Emrani A.S ${ }^{4}$., Esmaeily $H^{5}$., Taghipour $\mathrm{A}^{5}$., Mirhafez S.R ${ }^{6}$., Ferns G.A ${ }^{7}$., Mardan-Nik M ${ }^{3}$., Mohammadzadeh E ${ }^{8}$., Avan $\mathrm{A}^{8}$., Parichehr Hanachi ${ }^{9}$., Ghayour-Mobarhan $\mathbf{M}^{8}$.

\footnotetext{
${ }^{1}$ Department of Health Education and Health Promotion, School of Health, Social Determinants of Health Research Center, Mashhad University of Medical Sciences, Mashhad, Iran

${ }^{2}$ Department of Community Health, Faculty of Medicine and Health Sciences, University Putra Malaysia, Seri Kembangan, Malaysia

${ }^{3}$ Department of Modern Sciences and Technologies, School of Medicine, Mashhad University of Medical Sciences, Mashhad, Iran.

${ }^{4}$ Cardiovascular Research Center, School of Medicine, Mashhad University of Medical Sciences, Mashhad, Iran

${ }^{5}$ Department of Biostatistics \& Epidemiology, School of Health, Management \& Social Determinants of Health Research Center, Mashhad University of Medical Sciences, Mashhad, Iran

${ }^{6}$ Department of Basic Medical Sciences, Neyshabur University of Medical Sciences, Neyshabur, Iran

${ }^{7}$ Division of Medical Education, Brighton \& Sussex Medical School, Falmer, Brighton, Sussex BN1 9PH, UK

${ }^{8}$ Metabolic Syndrome Research Center, Mashhad University of Medical Sciences, Mashhad, Iran

${ }^{9}$ Department of Biology, Biochemistry Unit, Alzahra University, Tehran, IR, Iran
}

\section{Abstract}

Cytokines play a key role in the pathogenesis of coronary artery disease (CAD). The aim of current study was to investigate the relationship between the serum concentrations of 12 cytokines with mortality and extent of CAD in individuals undergoing angiography and healthy controls. $342 \mathrm{CAD}$ patients were recruited and divided into 2 groups: those with $\geq 50 \%$ occlusion in at least one coronary artery [Angiography (+)] or \&lt;50\% obstruction in coronary arteries [Angiography $(-)$ ]. Also 120 healthy subjects were enrolled as control group. Lipid profile, fasting blood glucose, body mass index, and blood pressure were evaluated in all the subjects. An Evidence Investigator ${ }^{\circledR}$ was used for measuring 12 cytokines (IL-1 $\alpha$, IL-1 $1 \beta$, IL-2, IL-4, IL-6, IL-8, IL-10, TNF- $\alpha$, MCP-1, IFN- $\gamma$, EGF, VEGF) using sandwich chemiluminescent assays. Univariate analysis, multivariate regression models, ROC, and Kaplan-Meier survival curves were used for exploring the candidate markers in CAD patients. Serum level of IFN- $\gamma$, IL-4, MCP-1, EGF, IL-6, and IL-8 were markedly higher in angiogram-positive patients, while VEGF concentrations were significantly (P \&lt; 0.05) lower, compared to control group. ROC analysis for MCP-1 showed that a cut-off of $61.95 \mathrm{pg} / \mathrm{mL}$ had $91 \%$ sensitivity and $91 \%$ specificity for predicting CAD patients. Moreover, \&gt;2.16 pg/mL IL-6 had a \&gt; $94 \%$ sensitivity and $70 \%$ specificity in predicting 2 years mortality in the subjects with a serum MCP$1 \& \mathrm{gt} ; 61.95 \mathrm{pg} / \mathrm{mL}$, and patients having IL-6/MCP-1 combination had a shorter survival.Our findings demonstrate that CAD patients with serum MCP-1 and IL-6 levels of \&gt;61.95 and $\& \mathrm{gt} ; 2.16 \mathrm{pg} / \mathrm{mL}$ had a higher mortality with $94.1 \%$ sensitivity and $70.5 \%$ specificity for predicting mortality in CAD patients.

Keywords: Cytokines, Coronary artery disease, Lipid profile, MCP-1/IL-6 